Document downloaded from:

http://hdl.handle.net/10251/43578

This paper must be cited as:

Romero García, V.; Picó Vila, R.; Cebrecos Ruiz, A.; Staliünas, K.; Sánchez Morcillo, VJ. (2013). Angular bandgaps in sonic crystals: evanescent waves and spatial complex dispersion relation. Journal of Vibration and Acoustics. 135(4):410121-410126. doi:10.1115/1.4023832

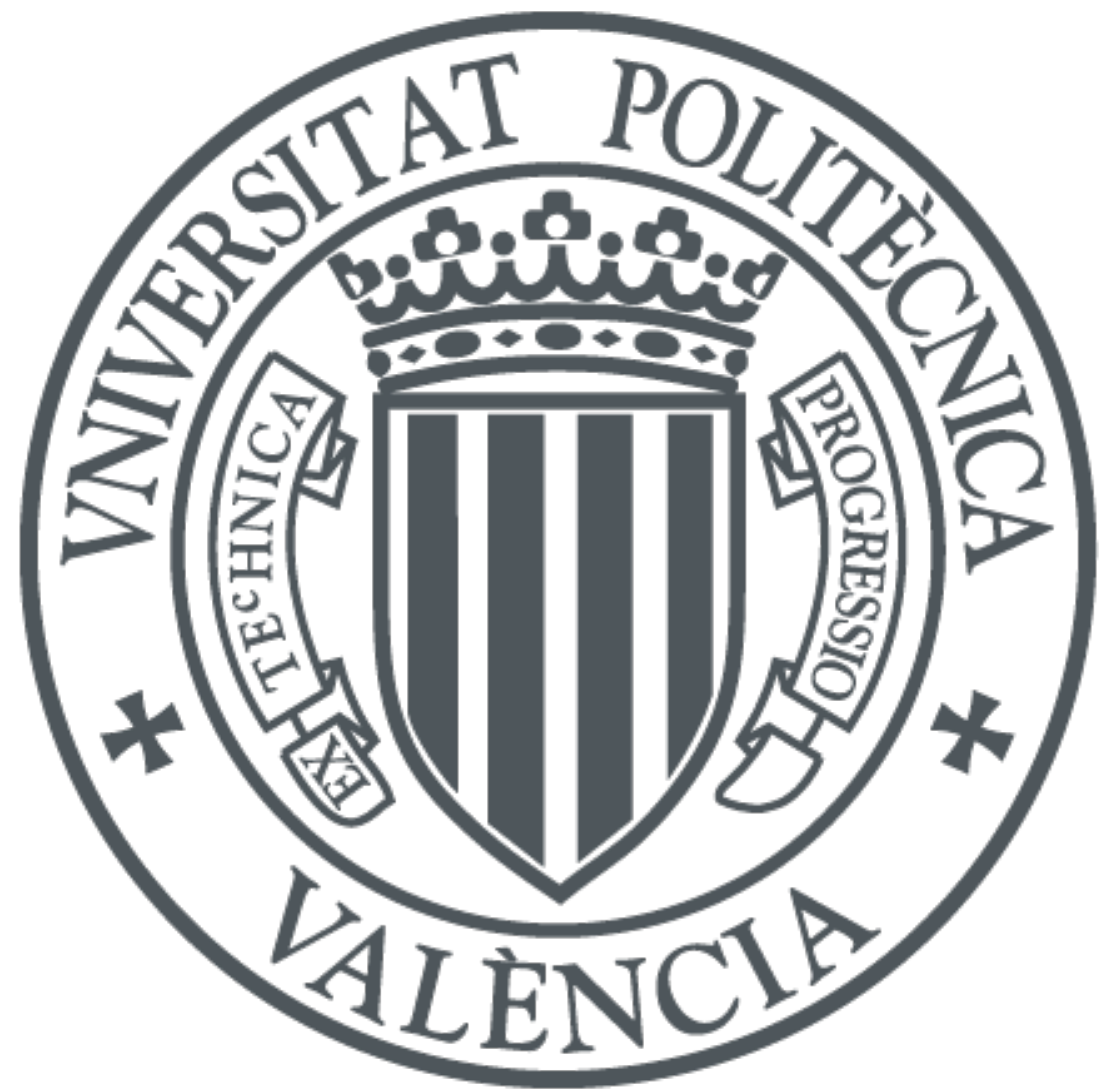

The final publication is available at

http://dx.doi.org/10.1115/1.4023832

Copyright American Society of Mechanical Engineers (ASME)

Additional Information 


\section{Angular Band Gaps in Sonic Crystal: Evanescent Waves and Spatial Complex Dispersion Relation}

\author{
V. Romero-García \\ Instituto de Investigación para la \\ Gestión Integrada de zonas Costeras \\ Universitat Politècnica de València \\ Paranimf 1, 46730, Gandia, Spain \\ Email: virogar1@gmail.com

\section{A. Cebrecos} \\ Instituto de Investigación para la \\ Gestión Integrada de zonas Costeras \\ Universitat Politècnica de València \\ Paranimf 1, 46730, Gandia, Spain \\ Email: alcebrui@epsg.upv.es
}

\author{
R. Picó
}

Instituto de Investigación para la Gestión Integrada de zonas Costeras Universitat Politècnica de València Paranimf 1, 46730, Gandia, Spain Email: rpico@fis.upv.es

\author{
K. Staliunas \\ Institució Catalana de Recerca \\ i Estudis Avançats (ICREA) \\ Pg. Lluis Companys, 23, \\ ES-08010, Terrassa, Spain
}

Email: kestutis.staliunas@icrea.es

\author{
V. J. Sánchez-Morcillo \\ Instituto de Investigación para la \\ Gestión Integrada de zonas Costeras \\ Universitat Politècnica de València \\ Paranimf 1, 46730, Gandia, Spain \\ Email: victorsm@fis.upv.es
}

Phononic crystals are artificial materials made of a periodic distribution of solid scatterers embedded into a solid host medium with different physical properties. An interesting case of Phononic Crystals, known as Sonic Crystals (SCs), appears when the solid scatterers are periodically embedded in a fluid medium. In SCs only longitudinal modes are allowed to propagate and both the theoretical and the experimental studies of the properties of the system are simplified without loss of generality. The most celebrated property of these systems is perhaps the existence of spectral band gaps. However, the periodicity of the system can also affect to the spatial dispersion, making possible the control of the diffraction inside these structures. In this work we study the main features of the spatial dispersion in SCs from a novel point of view taking into account the evanescent properties of the system, i.e., studying the complex spatial dispersion relations. The evanescent behaviour of the propagation of waves in the angular band gaps are theoretically and experimentally observed in this work. Both the numerical predictions and the experimental results show the presence of angular band gaps in good agreement with the complex spatial dispersion relation. The results shown in this work are independent of the spatial scale of the structure, and in principle the fundamental role of the evanescent waves could be also expected in micro- or nano-scale phononic crystals.

\section{Nomenclature \\ PC Phononic Crystal. \\ SC Sonic Crystal.}

PWE Plane wave expansion.

EPWE Extended plane wave expansion. MST Multiple scattering theory.

BG Band gap.

a Lattice constant.

$f$ Filling fraction.

$r$ Radius of the scatterer.

$\Psi \quad$ Normalized frequency.

SPL Sound pressure level.
$k$ Wave number
$v$ Frequency
$\rho$ Density
$\kappa \quad$ Bulk modulus
$\omega$ Angular frequency
c Sound velocity 


\section{Introduction}

The study of wave propagation in periodic media has a long history in the field of vibrations and acoustics [1-3]. In recent years, after the pioneering works of Yablonovitch [4] and John [5], who discovered simultaneously the possibilities to control the light flow in periodic distribution of dielectric materials, an increasing interest appeared in the analogous structures to control both the elastic and acoustic waves using the well-known phononic crystals (PC). Several theoretical works started the analysis of periodic arrays made of isotropic solids embedded in an isotropic elastic background [6-11]. By analogy with the photonic case, these periodic arrangements present acoustic band gaps (BGs), defined as frequency ranges where vibrations, sound and phonons are forbidden. A particular case of PC, is the Sonic Crystal (SC) $[12,13]$ which consist of solid scatterers embedded in a fluid host medium. In this case an important simplification without loss of generality arises in this system, which allows considering the propagation of only longitudinal waves. SC are specially relevant due to the experimental possibilities as for example measurements inside the crystal.

The measurements of the sound attenuation by a sculpture, desinged by Eusebio Sempere and exhibited at the Juan March Foundation in Madrid, constituted the first experimental evidence of the presence of BG in a SC [12]. The work of Martínez-Sala et al. [12] experimentally showed that the repetition of rigid cylinder rods (2D), inhibited the sound transmission for certain frequency ranges related to this periodic modulation, just as photonic crystals do with light. The subsequent theoretical predictions $[13,14]$ and experimental results [15] were motivated by these experimental results in order to explain the propagation properties of this sculpture that could filter noise.

Since these acoustical properties were measured in that minimalist sculpture, a great research interest, both experimental and theoretical, have been focused on the exploitation of the particular dispersion relation of these periodic structures revealing very interesting physical properties, showing a resurgence of fundamental and applied importance in condensed matter physics. The existence of complete elastic/acoustic BG, the possibilities of real applications such as elastic/acoustic filters [16], or even as noise control devices $[17,18]$, improvements in the design of transducers $[19,20]$, or testing fundamental pure physics phenomena [21-31] are several examples that motivated this growing interest.

The influence of the spatial periodicity on the spectral dispersion of such systems is represented by the spectral dispersion, however it has come out that the spatial periodicity can affect not only this temporal dispersion, but also the spatial one making possible the control of the diffraction inside the periodic structures. Due to that, one can observe different behaviour depending on the spatial dispersion relation, i.e., on the curvature of the isofrequency contours [32]. The socalled self-collimation effect, due to flat isofrequency contours, consists in the propagation of a beam in the periodic system without apparent diffraction keeping its original size. This phenomenon has been experimentally demonstrated to date at different ranges of frequency for both electromag- netic waves $[33,34]$ and acoustic waves $[35,36]$ in photonic and sonic crystals respectively. Oppositely, when the curvature of the isofrequency contour is negative, one can observe focusing due to the all angle negative refraction phenomenon, which has been also observed in both electromagnetics [37,38] and acoustics [39-41].

The previous spatial effects, the self-collimation and all angle negative refraction, occur for connected (continuous) isofrequency contours, however, there are frequencies in which the isofrequency lines are discontinuous in the Brillouin zone, making possible the existence of angles in which no isolines exist and, as a consequence, there is not propagation of waves. These ranges of angles are called angular band gaps [32,35].

In this work we introduce the imaginary part of the relation dispersion, traditionally not considered, to theoretically and experimentally interpret the spatial dispersion in $\mathrm{SC}$, specially to interpret the presence of the angular band gaps. From the complex dispersion relation, $k(\omega)$, we pay attention to the existence of evanescent waves along the angles in which angular band gaps are predicted. Novel representation of the complex isofrequency curves, i.e. the dependence of the real and imaginary longitudinal component of the propagation constant versus the real and imaginary transverse component, $k_{\|}=k_{\|}\left(k_{\perp}\right)$, are introduced in this work to interpret the angular band gaps. Experimental results, in good agreement with the predictions of both the multiple scattering and complex dispersion relation, are also shown here.

This work is organized as follows. First of all, in Section 2, we introduce the complex dispersion relation, $k(\omega)$. We will show the procedure to represent the complex isofrequency contours (spatial complex dispersion relation), paying attention to the case analyzed in this work in which angular band gaps appear. After that, Section 3 shows the experimental set up we use to analyze the angular band gaps. Section 4 shows the numerical and the experimental evidences of the angular band gaps, but also, in order to be a selfconsistent work the several effects of the spectral dispersion are also briefly summarized. The concluding remarks are shown in Section 5.

\section{$2 k(\omega)$ complex relation dispersion}

Due to the periodicity of the system, the relation dispersion has been traditionally obtained from solving the following eigenvalue problem using the plane wave expanssion (PWE) procedure [10]

$$
\sum_{\vec{G}^{\prime}}\left((\vec{k}+\vec{G}) \sigma_{k}\left(\vec{G}-\vec{G}^{\prime}\right)\left(\vec{k}+\vec{G}^{\prime}\right)-\omega^{2} \eta_{\vec{k}}\left(\vec{G}-\vec{G}^{\prime}\right)\right) p_{\vec{k}}\left(\vec{G}^{\prime}\right)=0 .
$$

For $\vec{G}$, being the reciprocal vector and taking all the possible values, Equation (1) constitutes a set of linear, homogeneous equations for the eigenvectors $p_{\vec{k}(\vec{G})}$ and the eigenfrequencies $\omega(\vec{k})$. However, this relation dispersion, $\omega(\vec{k})$ does not take 

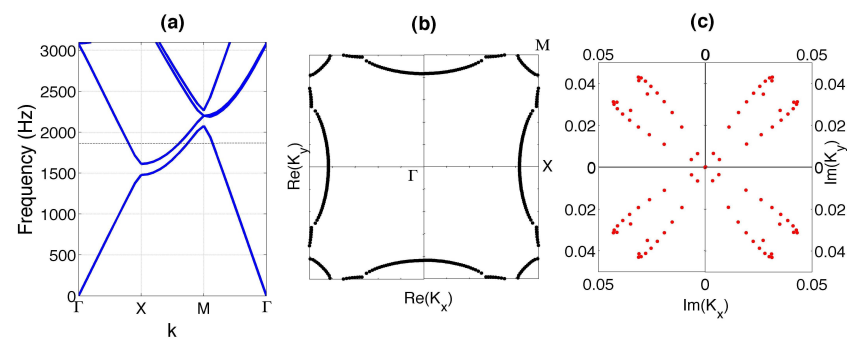

Fig. 1. Analysis of the eigenvalue problem using EPWE. (a) Real part of the complex band structure for a square array of rigid cylinders embedded in air. Horizontal dashed line represents the studied frequency. Real (b) and imaginary (c) parts of the isofrequency contours at $\mathrm{V}$. Analysis of the scattering problem at the selected frequency.

into account the evanescent waves, as only real values can be obtained.

However, if one solve the inverse problem, $k(\omega)$, the solutions are not necessarily forced to be real, and they could be also complex, introducing the evanescent waves in the interpretation of the dispersion relation of Sonic Crystals [42,43]. The inverse problem can be formulated in terms of the Extended Plane Wave Expansion (EPWE) as [44]

$$
\left(\begin{array}{cc}
\omega^{2} \Omega-\sum_{i=1}^{3} \Gamma_{i}^{0} \Sigma \Gamma_{i}^{0} & 0 \\
-\sum_{i=1}^{3} \Sigma \Gamma_{i}^{0} & I
\end{array}\right)\left(\begin{array}{c}
P \\
\Phi^{\prime}
\end{array}\right)=k\left(\begin{array}{cc}
\sum_{i=1}^{3} \Gamma_{i}^{0} \Sigma \alpha_{i} & I \\
\sum_{i=1}^{3} \Sigma \alpha_{i} & 0
\end{array}\right)\left(\begin{array}{c}
P \\
\Phi^{\prime}
\end{array}\right)
$$

This equation represents a generalized eigenvalue problem with $2 N$ eigenvalues $k$. The complex spectral relation dispersion in a incidence direction $\vec{\alpha}$ can be obtained by solving the eigenvalue equation for a discrete number of frequencies and then sorted by continuity of $k$. However the complex spatial dispersion can be obtained by solving the problem for a fixed frequency in all the incident directions.

Figure 1(a) shows the real part of the band structure for the SC we are dealing with in this work. We consider a SC with square periodicity, which lattice constant is, $a=11 \mathrm{~cm}$. In this case the filling fraction is $f=0.1$. Along the work we will use the normalized units, therefore the normalized frequency is $\Psi=v a / c_{\text {host }}$. The horizontal dashed line, represents the frequency under study in this work. The imaginary part of the complex band structures has been recently exploited to obtain the evanescent properties for the spectral dispersion relation in SC $[27,31,42,44-46]$. In this work we are not interested in the imaginary part of the spectral dispersion relation but in the imaginary part of the spatial dispersion relation. Figures 1(b) and 1(c) show the real and the imaginary parts of the isofrequency contours at the frequency under study respectively. We notice that imaginary isofrequency curves appear for the ranges of angles where no real isofrequency lines are predicted. As a consequence, an angular BG appears from the real part of the isofrequency contours and its evanescent behaviour is characterized by the imaginary part. It is worth noting that the real part of the spatial dispersion relation obtained using EPWE, coincides with the real part obtained using PWE.
Due to the presence of this imaginary part of the isofrequency contours, an exponential decay appear at this angles which decay rate depends on the imaginary part of the value of the wave vector (of the isofrequency contours). Thus, one can expect for finite structures a dependence of the attenuation properties at the angular BG on the number of rows of the $\mathrm{SC}$, as will be discussed bellow.

\section{Experimental Setup}

All the experimental results shown in this work have been measured under controlled conditions in an anechoic chamber. The analyzed SC is made of aluminum cylinders embedded in air, with the same filling fraction as in the theoretical predictions shown in Section 4.

All the acoustic measurements were recorded using a free-field microphone 1/2" Type 4189 B\&K. Thes microphone was controled by a 3D Robotized e-Acoustic Measurement System (3DReAMS), which is a Cartesian robot with three axes $(\mathrm{X}, \mathrm{Y}, \mathrm{Z})$ installed in the ceiling of the anechoic chamber. The robot was designed to sweep the microphone through a 3D grid of measuring points located at any trajectory inside the echo-free chamber. The robot includes a rotatory column installed on the ceiling of the anechoic chamber, where the periodic arrays are hung in a frame [47].

The National Instruments cards PCI-4474 and NI PCI7334 together with the Sound and Vibration Toolkit and the Order Analysis Toolkit for LabVIEW were used for both the data acquisition and the motion of the robot. Once the robotized system is turned off and both the acoustic source and the microphone are turned on, the microphone acquires the temporal signal. From this temporal signal, one can obtain the power spectra, the frequency response or the sound-level measurement.

\section{Spatial complex dispersion}

In modulated materials waves acquire different phases depending on the angles of propagation inside the periodic system because the curvature of the complex isofrequency contours depends on the propagating angle. This is the milestone of the geometric interpretation of the spatial dispersion. The geometrical interpretation of wave diffraction is as follows: wave beams of arbitrary shape can be Fourier decomposed into plane waves, which in propagation acquire phase shifts depending on their propagation angles. This dephasing of the plane-wave components results in a diffractive broadening of the beams. Figure 2(a) illustrates normal diffraction in propagation through an homogeneous material, where the longitudinal component of the wave vector depends trivially on the propagation angle. In general, the normal or positive diffraction means that the surfaces of constant frequency are concave in the wave vector domain as illustrated in Fig. 2(a). Negative diffraction, as illustrated in Fig. 2(b), geometrically implies that the surfaces of constant frequency are convex in the wave vector domain. The intermediate case of vanishing diffraction is illustrated in Fig. 2(c), where zero diffraction is supposed to occur at a particular point in $k$-space where 
(a)
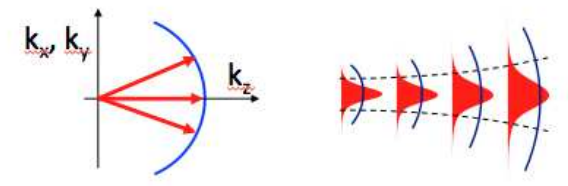

(b)

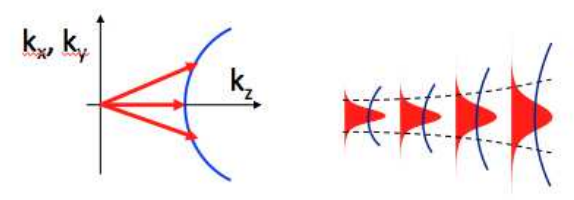

(c)

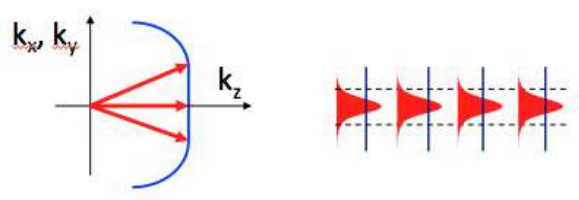

(d)

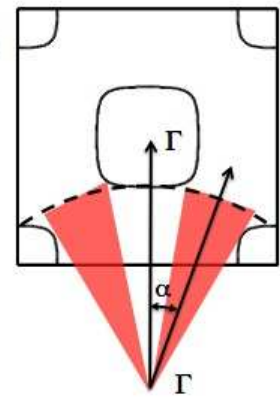

Fig. 2. Geometrical interpretation of diffraction of waves propagating along the $z$ axis: (a) positive, or normal diffraction in propagation through homogeneous materials; (b) negative, or anomalous diffraction; (c) zero diffraction. The area of negligible diffraction (for evaluation of the minimum size of the nondiffractive beam) is indicated. Reference [35]. (d) Different regimes considered: $a$ broad beams with spatial spectra inside the parabolic area of the spatial dispersion curve, $b$ beams of intermediate width, with spatial spectra filling the full width of the isoline of the given band, $c$ narrow beams with the spatial spectra extending over isolines from the neighboring bands, and thus overlapping the band gaps in angular domain. The region denoted by $d$ corresponds to the forbidden angles (band gaps in space spectra domain). (d) Schematic representation of the angular BG.

the curvature of the surfaces of constant frequency becomes exactly zero. Zero diffraction physically means that beams of arbitrary width can propagate without diffractive broadening. Therefore, the comprehensive study of the isofrequency surfaces provide crucial information to properly understand diffraction inside crystals for sound frequencies outside the BG. This allows the management of spatial dispersion i.e., the diffraction properties of narrow beams.

\subsection{Negative curvature. All Angle Negative Refraction}

A negative refraction index would allow a flat slab of a material to behave with special properties as for example a left handed material or as a lens. Concerning periodic structures, one can achieve negative refraction in two different ways. The first way is based on the double negativity of the effective parameters $[48,49]$ in metamaterials. The other way arises from the negative curvature of the isofrequency contours $[37,38]$, in which the periodic structure have an effective refractive index controlled by the band structures. The properties of the $\mathrm{SC}$ in the range of frequencies above the first BG, where the wavelengths are much smaller than the lattice constant in SC, were used by Yang et al. [39] to introduce the negative refractive index in the field of the SC. The authors claim that the relationship between the phase velocity and the wave vector in the second band suggests that both focusing and large negative refraction phenomena may occur. The negative refraction behavior and imaging effect in periodic structures have been also experimentally observed [40]. Negative-refraction and imaging effects of surface water waves by a periodic structure were also theoretically and experimentally demonstrated recently [50]. The anomalous features of negative refraction open the door to a variety of applications.

It has been observed that one can also obtain imaging without negative refraction index. At the end of the first band, near the band edge, the band becomes curved and, as a consequence, group velocity inside the crystal is a function of frequency. At this stage the spatial dispersion relation is relevant. At these frequencies all-angle negative refraction has been observed without negative effective index around the $\Gamma \mathrm{M}\left(45^{\circ}\right)$ direction. At the $\mathrm{M}$ point, the curvature of the isofrequency contour $\left(\partial^{2} \omega / \partial k_{i} \partial k_{j}\right)$ is negative, i.e., the contour is convex and represents inward-pointing group velocities. As a consequence, for frequencies corresponding to allconvex contours, negative refraction occurs. Then, negative refraction can be observed also in the first band where neither a negative group velocity nor a negative effective index is a prerequisite for negative refraction. The phenomenon of the all-angle negative refraction in SC was observed, showing a strong dependence on the frequency and on the incidence angles [41].

\subsection{Flat isofrequency contours: Self-collimation or zero diffraction.}

The propagation of waves through a periodic system is mainly characterized by dispersion, leading to a strong scattering of the waves in multiple directions, but there is a fascinating effect, originally named self-collimation or subdiffraction in which a beam propagates in the periodic system without apparent diffraction keeping its original size. Usually, this phenomenon appears at high propagating bands, where flat isofrequency contours can be obtained. This phenomenon has been experimentally demonstrated to date for different frequency ranges of electromagnetic waves, in particular in the optical [33] and microwave [34] regimes. In the acoustic counterpart, subdiffractive propagation of sonic waves in phononic (or sonic) crystals was reported to occur in 2D SC's $[35,36]$. It is worth noting that this subdiffractive sonic beams are supported by crystals with perfect symmetry, therefore do not require the presence of defects, different from other waveguiding phenomena previously reported. Experimental realization of the acoustic selfcollimation of an ultrasonic beam inside a three-dimensional 


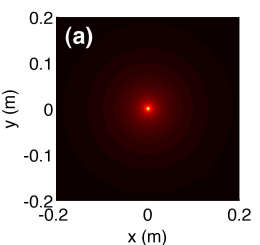

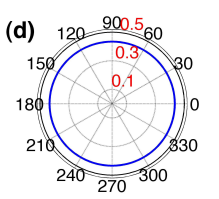
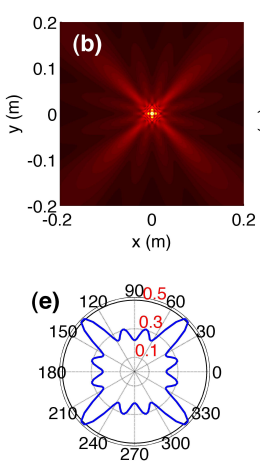
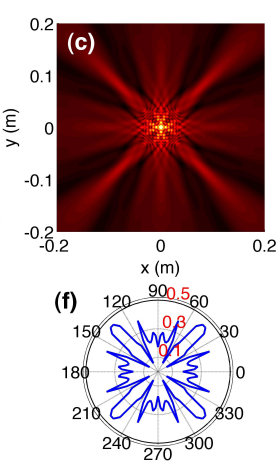

Fig. 3. Analysis of the scattering problem using multiple scattering theory. (a), (b) and (c) represent the pattern of the absolute value of the pressure for a linear source, a linear source inside (in the middle of the SC) a $6 \times 6$ PC and a linear source inside a $16 \times 16$ PC respectively. (d), (e) and (f) show the polar profiles of the absolute value of the pressure for different radial distances from the source in each SC.

3D sonic crystal was also reported. The crystal is formed by two crossed steel cylinders structures in a woodpilelike geometry disposed in water. Measurements of the 3D field distribution show that a narrow beam, which diffractively spreads in the absence of the sonic crystal, is strongly collimated in propagation inside the crystal, demonstrating the 3D self-collimation effect.

\subsection{Angular band gaps.}

In addition to BG in the band structure (spectral dispersion relation), the periodic structure can also modify the spatial dispersion, allowing the managing of the diffractive broadening of beams $[32,35,36]$. The interaction of the spatial spectrum of the incident wave with the isofrequency curves of the modulated material can produce different focusing regimes depending on the curvature of the isolines in $\vec{k}$-space, one example is the self-collimation discussed above. On the other hand, there are angles in which no isolines exist in the $\vec{k}$-space and this results in angular BGs.

Due to the fact that there are no isolines for certain angles, no projection of the wave vector of the incident wave on the isofrequency line is achieved. As a consequence, propagation is not allowed at these angles for a given frequency. These angular BGs have been usually interpreted with the classical isofrequency lines obtained from the $\omega(\vec{k})$ relations. Figure 2(d) shows an schematic interpretation of the angular BG. There are frequencies in which the wave vectors of the incident wave do not reach any isofrequency contour, therefore this wave vector cannot excite Bloch mode. The region covered by red area in Figure 2(d) corresponds to this situation, and it represents the forbidden angles (BGs in space spectra domain), which constitutes the interest of this Section.

As in the case of spectral BGs, angular BGs exist because of the presence of evanescent waves. In this Section we study these evanescent waves. We solve the inverted problem $k(\omega)$ and we compare both theoretically and experimentally
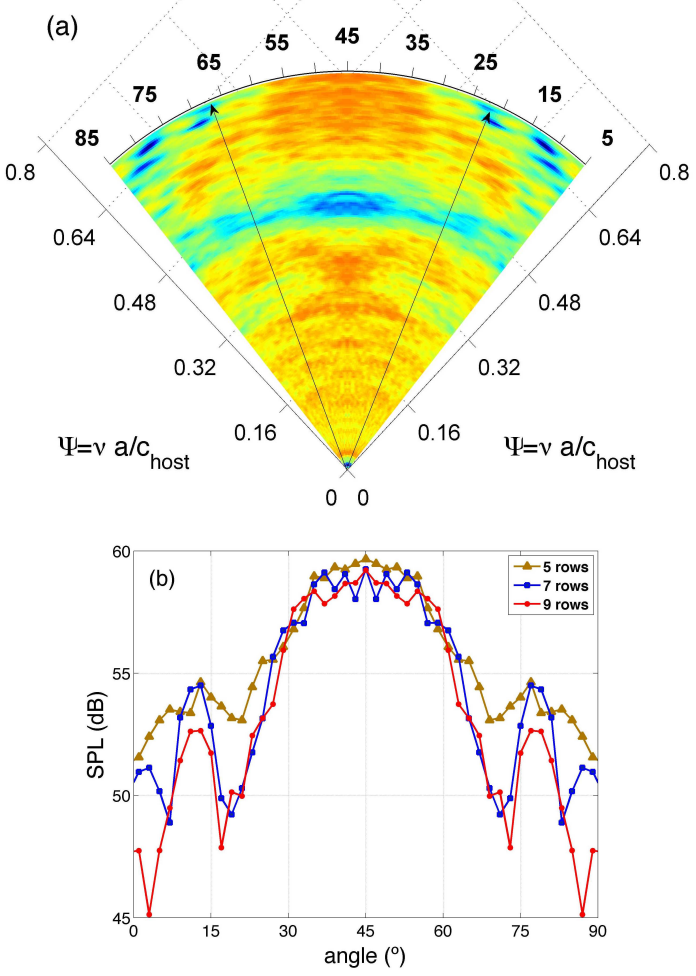

Fig. 4. Experimental analysis of the angular band gaps. We have analyzed three cases: 5, 7 and 9 rows. (a) Experimental polar map of the sound pressure level spectrum for the case of 9 rows. Color scale represents the sound pressure level. (b) Cuts at the analyzed frequency for the three analyzed cases.

the values of the scattering problem in finite structures. The eigenvalue problem is solved using the EPWE $[42,43,46,51]$ and the scattering problem is solved using the multiple scattering theory $[52,53]$. We obtain complex isofrequency contours at angles in which angular BGs are predicted. As we have previously discussed, due to the complex value of the wavevector in periodic media, the isofrequency contours can be represented in terms of the real and imaginary part of $\vec{k}$. The real part is related with the propagating properties of the wave whereas the imaginary part is related with the evanescent properties.

We start with the analysis of the finite structures using multiple scattering theory. Figures 3(a), 3(b) and 3(c) show the maps of absolute value of the pressure (amplitude) at $\Psi=$ 0.58 for three different cases: (a) an isolated line source, (b) a line source in the middle of a $6 \times 6 \mathrm{SC}$ and (c) a line source in the middle of a $16 \times 16 \mathrm{SC}$ respectively. For these three cases we show in 3(d), 3(e) and 3(f) the polar plots. Note that, as we increase the number of rows, there are angles at which the attenuation increases. This can be interpreted by the presence of evanescent waves in such directions.

Now we recover the isofrequency contours obtained in Section 2. We pay special attention to the imaginary part of the spatial dispersion relation. At some given directions evanescent lobes appears, corresponding to angles in which attenuation occurs. Due to the presence of evanescent waves, attenuation is expected to increase as the number of rows in 
the SC increases.

The next step consist of comparing both Fig. 1(c) with the insets in Fig. 3. On one hand we observe that for angles at which an evanescent lobe is predicted, a strong amplitude decrease at the polar plot appears. Moreover the larger the number of rows the deeper the trough in amplitude. On the other hand, we can also observe a focused beam at $\Gamma \mathrm{M}$ direction, due to the convexity of the isofrequency contours at point $\mathrm{M}$ (as we have explained in the previous Section). Therefore, at $\Gamma \mathrm{M}$ direction a focused beam appears but around this focused beam, one can clearly see the angular BGs.

In order to experimentally assess the presence of evanescent waves at different directions of propagation, we use a 2D SC composed by a square lattice of cylindrical scatterers truncated in a triangular outer shape. The source is oriented at the $\Gamma \mathrm{M}$ direction $\left(45^{\circ}\right)$ of the crystal. The microphone positions are controlled by 3DReAMs (see Section 3 ) in order to measure at the points located in a circular trajectory around the SC. These positions correspond to different directions of propagation in the crystal.

The experimental polar maps of the values of pressure for the case of a SC made of 9 rows is shown in Fig. 4(a). This polar map represents the frequency response of a finite periodic array. Each radial line represents the sound pressure level (SPL) spectrum for a given incidence angle. Normalized units with respect to both the lattice constant and the host material are used in the representation $\left(\Psi=v a / c_{\text {host }}\right)$. It is worth to highlight the complexity shown in the polar map of the finite structure and how we can identify for example the spectral band gap due to the spectral dispersion relation. But our interest in this work is focused on the spatial dispersion relation. In this sense, one can observe the effect of the evanescent behaviour in the direction where an angular band gap is predicted by theory (shown by black arrows). Three different configurations have been measured by removing rows of scatterers in the crystal: 5 rows, 7 rows and 9 rows. The angular cuts of SPL for these three cases are shown in Fig. 4(b) using red continuous line with red circles, blue continuous line with blue square and brown continuous line with brown triangles respectively. One can see the troughs in the sound pressure level in the position of the angular BGs. On the other hand, the decreasing of the values of SPL at this directions as we increase the number of rows is a clear effect of the presence of evanescent waves. Thus, experimental results show that there is a close relation between evanescent waves and the presence of the angular (spatial) filtering.

\section{Conclusions}

The presence of the evanescent waves has been found of fundamental interest to theoretically and experimentally interpret phenomena based on the spatial complex relation dispersion, such us the presence of angular band gaps. Then, both the propagating (real part of the complex relation dispersion) and the evanescent (imaginary part) properties of these systems are necessary to explain the control of the diffraction and the diffusion of acoustic waves with periodic structures. Finally, the properties of these systems are independent of the spatial scale of the structure, and as a consequence the control of phonons by means of periodic systems could be a promising area [54-57]. Therefore, in principle all phenomena could be observed in micro- or nano-scale phononic (so called hypersonic [58]) crystals, and evanescent waves can play an important role. At these scales phonons are intimately related to thermal effects and the ideas presented in this work could find relevance also for heat management in acoustical or acousto-optical devices.

\section{Achnowledgements}

This work was supported by MCI Secretaría de Estado de Investigación (Spanish government) and the FEDER funds, under grant MAT2009-09438, FIS2011-29734-C0202 , and from Generalitat Valencia through the project GV/2011/055. VRG is grateful for the support of "Programa de Contratos Post-Doctorales con Movilidad UPV (CEI-0111)". We acknowledge the Centro de Tecnologías Físicas: Acústica, Materiales y Astrofísica and the Sonic Crystal Technologies Research Group of the Universitat Politècnica de València for the use of the anechoic chamber and the 3DReAMS respectively.

\section{References}

[1] Rayleigh, J., 1887. "Acoustical observations". Philosophical Magazine, XXIV, pp. 145-159.

[2] Bloch, F., 1928. "Uber die quantenmechanik der electron in kristallgittern". Zeifschrift fr Physik, 52, pp. 555-600.

[3] Brillouin, L., 1953. Wave Propagation in Periodic Structures, 2nd. ed. Dover, New York.

[4] Yablonovitch, E., 1987. "Inhibited spontaneous emission in solid-state physics and electronics". Phys. Rev. Lett., 58, p. 2059.

[5] John, S., 1987. "Strong localization of photons in certain disordered dielectric superlattices". Phys. Rev. Lett., 58, p. 2486.

[6] Ruffa, A., 1992. "Acoustic wave propagation through periodic bubbly liquids". J. Acoust. Soc. Am., 91, pp. 16.

[7] Sigalas, M., and Economou, E., 1992. "Elastic and acoustic wave band structure". J. Sound Vib., 158, p. 377.

[8] Sigalas, M., and Economou, E., 1993. "Band structure of elastic waves in two dimensional systems". Solid State Commun., 86, p. 141.

[9] Kushwaha, M., Halevi, P., Dobrzynski, L., and DjafariRouhani, B., 1993. "Acoustic band structure of periodic elastic composites". Phys. Rev. Lett., 71(13), pp. 20222025.

[10] Kushwaha, M., Halevi, P., Martnez, G., Dobrzynski, L., and Djafari-Rouhani, B., 1994. "Theory of acoustic band structure of periodic elastic composites". Phys. Rev. B, 49 (4)(4), pp. 2313-2322. 
[11] Sigalas, M., Economou, E., and Kafesaki, M., 1994. "Spectral gaps for electromagnetic and scalar weaves: Possible explanation for certain differences". Phys. Rev. B, 50, p. 3393.

[12] Martínez-Sala, R., Sancho, J., Sánchez, J. V., Gómez, V., Llinares, J., and Meseguer, F., 1995. "Sound attenuation by sculpture". Nature, 378, p. 241.

[13] Sánchez-Pérez, J. V., Caballero, D., Martínez-Sala, R., Rubio, C., Sánchez-Dehesa, J., Meseguer, F., Llinares, J., and Gálvez, F., 1998. "Sound attenuation by a twodimensional array of rigid cylinders". Phys. Rev. Lett., 80(24), pp. 5325-5328.

[14] Kushwaha, M., 1997. "Stop-bands for periodic metallic rods: Sculptures that can filter the noise". Appl. Phys. Lett., 70, p. 3218.

[15] Robertson, W. M., and RudyIII, J. F., 1998. "Measurement of acoustic stop bands in two-dimensional periodic scattering arrays". J. Acoust. Soc. Am., 104(2), pp. 694-699.

[16] Khelif, A., Choujaa, A., Djafari-Rouhani, B., Wilm, M., Ballandras, S., and Laude, V., 2003. “Trapping and guiding of acoustic waves by defect modes in a full-band-gap ultrasonic crystal". Phys. Rev. B, 68, p. 214301.

[17] Sánchez-Pérez, J., Rubio, C., Martínez-Sala, R., Sánchez-Grandia, R., and Gómez, V., 2002. "Acoustic barriers based on periodic arrays of scatterers". Appl. Phys. Lett., 81, p. 5240.

[18] Romero-García, V., and J.V. Sánchez-Pérez, L. G.R., 2011. "Tunable wideband bandstop acoustic filter based on two-dimensional multiphysical phenomena periodic systems". J. Appl. Phys., 110, p. 014904.

[19] Qiu, C., Liu, Z., Shi, J., and Chan, C. T., 2005. "Directional acoustic source based on the resonant cavity of two-dimensional phononic crystals". Appl. Phys. Lett., 86, p. 224105.

[20] Qiu, C., and Liu, Z., 2006. "Acoustic directional radiation and enhancement caused by band-edge states of two-dimensional phononic crystals". Appl. Phys. Lett., 89, p. 063106.

[21] Sigalas, M., 1998. "Defect states of acoustic waves in a twodimensional lattice of solid cylinders". J. Appl. Phys., 84, p. 3026.

[22] Tanaka, Y., Yano, T., and ichiro Tamura, S., 2007. "Surface guided waves in two-dimensional phononic crystals". Wave Motion, 44, pp. 501-512.

[23] Vasseur, J. O., Deymier, P. A., Djafari-Rouhani, B., Pennec, Y., and Hladky-Hennion, A.-C., 2008. "Absolute forbidden bands and waveguiding in twodimensional phononic crystal plates". Phys. Rev.B, 77, p. 085415.

[24] Zhao, Y., and L.B.Yuan, 2009. "Characteristics of multi-point defect modes in 2 s phononic crystals". $J$. Phys. D: Appl. Phys., 42, p. 015403.

[25] Wu, L., Chen, L., and Liu, C., 2009. "Experimental investigation of the acoustic pressure in cavity of a two dimensional sonic crystal". Physica B, 404, p. 1766.

[26] Hussein, M., 2009. "Theory of damped bloch waves in elastic media". Phys. Rev. B, 80, p. 212301.

[27] Romero-García, V., Vasseur, J. O., Hladky-Hennion, A. C., Garcia-Raffi, L. M., and Sánchez-Pérez, J. V., 2011. "Level repulsion and evanescent waves in sonic crystals". Phys. Rev. B, 84, p. 212302.

[28] Farzbod, F., and Leamy, M., 2011. "Analysis of bloch's method in structures with energy dissipation". Jour. Acos. Vib, 133, p. 051010.

[29] Laude, V., Moiseyenko, R., Benchabane, S., and and, N. D., 2011. "Bloch wave deafness and modal conversion at a phononic cyrstal boundary". AIP Advances, 1, p. 041402.

[30] Moiseyenko, R., Herbison, S., Delercq, N., and Laude, V., 2012. "Phononic crystal diffraction grattings". Jour. Appl. Phys., 111, p. 034907.

[31] Romero-García, V., Vasseur, J., Garcia-Raffi, L., and Hladky-Hennion, A.-C., 2012. "Theoretical and experimental evidence of level repulsion states and evanescent modes in sonic crystal stubbed waveguides". New J. Phys., 14, p. 023049.

[32] Sánchez-Morcillo, V., Staliunas, K., Espinosa, V., Pérez-Arjona, I., Redondo, J., and Soliveres, E., 2009. "Propagation of sound beams behind sonic crystals". Phys. Rev. B, 80, p. 134303.

[33] Rakich, P. T., Dahlem, M. S., Tandon, S., Ibanescu, M., Soljai, M., Petrich, G. S., Joannopoulos, J. D., Kolodziesjski, L. A., and Ippen, E. P., 2006. “Achieving centimeter-scale super-collimation in ultra large area photonic crystals". Nat. Mater., 5, p. 93.

[34] Lu, Z., Shi, S., Murakowski, A., Schneider, G. J., Schuetz, C. A., and Prather, D. W., 2006. "Experimental demonstration of self-collimation inside a threedimensional photonic crystal". Phys. Rev. Lett., 96, p. 173902.

[35] Pérez-Arjona, I., Sánchez-Morcillo, V. J., Redondo, J., Espinosa, V., and Staliunas, K., 2007. "Theoretical prediction of the nondiffractive propagation of sonic waves through periodic acoustic media". Phys. Rev. B, 75(014304), p. 014304.

[36] Espinosa, V., Sánchez-Morcillo, V. J., Staliunas, K., Pérez-Arjona, I., and Redondo, J., 2007. "Subdiffractive propagation of ultrasound in sonic crystals". Phys. Rev. B, 76, p. 140302(R).

[37] Luo, C., Johnson, S. G., Joannopoulos, J. D., and Pendry, J. B., 2002. "All-angle negative refraction without negative effective index". Phys, 65, p. 201104(R).

[38] Luo, C., Johnson, S. G., Joannopoulos, J. D., and Pendry, J. B., 2003. "Subwavelength imaging in photonic crystals". Phys. Rev B, 68, p. 045115.

[39] Yang, S., Page, J. H., Liu, Z., Cowan, M. L., Chan, C., , and Sheng, P., 2004. "Focusing of sound in a 3d phononic crystal”. Phys. Rev. Lett., 93(2), p. 024301.

[40] Ke, M., Liu, Z., Qiu, C., Wang, W., Shi, J., Wen, W., and Sheng, P., 2005. "Negative-refraction imaging with two-dimensional phononic crystals". Phys. Rev. B, 72, p. 064306.

[41] Feng, L., Liu, X., Chen, Y., Huang, Z., Mao, Y., Chen, Y., Zi, J., and Zhu, Y., 2005. "Negative refraction 
of acoustic waves in two-dimensional sonic crystals". Phys. Rev. B, 72, p. 033108.

[42] Romero-García, V., Sánchez-Pérez, J., and GarciaRaffi, L., 2010. "Evanescent modes in sonic crystals: Complex dispersion relation and supercell approximation”. J. Appl. Phys., 108, p. 044907.

[43] Laude, V., Achaoui, Y., Benchabane, S., and Khelif, A., 2009. "Evanescent bloch waves and the complex band structure of phononic crystals". Phys. Rev. B, 80, p. 092301.

[44] Romero-García, V., Sánchez-Pérez, J., and GarciaRaffi, L., 2010. "Propagating and evanescent properties of double-point defects in sonic crystals". New. Jour. Phys., 12, p. 083024.

[45] Romero-García, V., Sánchez-Pérez, J., neira Ibáñez, S. C., and Garcia-Raffi, L., 2010. "Evidences of evanescent bloch waves in phononic crystals". Appl. Phys. Lett., 96, p. 124102.

[46] Romero-García, V., Garcia-Raffi, L. M., and SánchezPérez, J. V., 2011. "Evanescent waves and deaf bands in sonic crystals". AIP Advances, 1, p. 041601.

[47] Romero-García, V., 2010. "On the control of propagating acoustic waves in sonic crystals: analytical, numerical and optimization techniques". PhD thesis, Instituto de Cienca de Materiales de Madrid (CSIC), Universitat Politècnica de València.

[48] Li, J., and Chan, C., 2004. "Double-negative acoustic metamaterial". Phys. Rev. E, 70, p. 055602.

[49] Guenneau, S., Movchan, A., Ptursson, G., and Ramakrishna, S. A., 2007. "Acoustic metamaterial for sound focusing and confinement". New journal of Physics, 9, p. 399.

[50] Farhat, M., Guenneau, S., Enoch, S., Tayeb, G., Movchan, A. B., and Movchan, N. V., 2008. "Analytical and numerical analysis of lensing effect for linear surface water waves through a square array of nearly touching rigid square cylinders". Phys. Rev. E, 77, p. 046308.

[51] Hsue, Y., Freeman, A., and Gu, B., 2005. "Extended plane-wave expansion method in three-dimensional anisotropic photonic crystals". Phys. Rev B, 72, p. 195118.

[52] Martin, P., 2006. Multiple Scattering. Interaction of Time-Harmonic Waves with $N$ Obstcles. Cambirdge University Press, UK.

[53] Chen, Y. Y., and Ye, Z., 2001. "Theoretical analysis of acoustic stop bands in two-dimensional periodic scattering arrays". Phys. Rev. E, 64, p. 036616.

[54] McGaughey, A. J. H., Hussein, M. I., Landry, E. S., Kaviany, M., and Hulbert, G. M., 2006. "Phonon band structure and thermal transport correlation in a layered diatomic crystal". Phys. Rev. B, 74, p. 104304.

[55] Landry, E. S., Hussein, M. I., and McGaughey, A. J. H., 2008. "Complex superlattice unit cell designs for reduced thermal conductivity”. Phys. Rev. B, 77, p. 184302.

[56] Hopkins, P. E., Reinke, C. M., Su, M. F., III, R. H. O., Shaner, E. A., Leseman, Z. C., Serrano, J. R., Phinney,
L. M., and El-Kady, I., 2011. "Reduction in the thermal conductivity of single crystalline silicon by phononic crystal patterning”. Nano Lett., 11, p. 107.

[57] Davis, B. L., and Hussein, M. I., 2011. "Thermal characterization of nanoscale phononic crystals using supercell lattice dynamics". AIP Advances, 1, p. 041701.

[58] Gorishnyy, T., Ullal, C. K., Maldovan, M., Fytas, G., , and Thomas, E. L., 2005. "Hypersonic phononic crystals". Phys. Rev. Lett., 94, p. 115501. 